\title{
Selling Science to the MTV Generation
}

At Argonne National Laboratory, interaction with local high schools and universities is a high priority. During the summer months, students and teachers are hired for eight- to twelve-week periods and exposed to the world of research and development. Last summer, our section hired Ken Helberg, a local high school science teacher who was assigned to work with me. We worked together quite well, and late that summer Ken asked me to come and speak to his classes. I put off saying yes for a time, but then read a survey of high school students who were asked what careers they would choose. To my surprise, more than $50 \%$ said they would like to be lawyers! Perhaps they were influenced by television's glamorous portrayals of wealth.

At that point, I decided that it was time to do a commercial about the joy of science and the exhiliration of discovering the unknown. I thought of the special events that had steered me to a career in science, and reasoned that perhaps I could present science in such a way as to add a few more scientists to the next survey. So I finally agreed, but rather than give presentations to six groups of $50 \mathrm{stu}$ dents each, I requested that the classes be combined. Thus my challenge was to inspire 300 freshman high school physics students.

The simplicity of Newton's laws, which can explain complex events such as a trip to the moon, was my chosen topic. The goal was to apply Newton's laws to everyday events. My introduction posed the question of what Newton's laws have to say about getting out of bed in the morning. From there, I covered Newton's three laws with my own interpretations. I also included the first two laws of thermodynamics.

Rather than use the conventional lecturing approach, I asked for volunteers. There were many, and I found that the attention-starved students all wanted to be involved in the show and that my presentation format gave them what they wanted. As a bonus, I discovered that I held more of the audience's attention when friends watched to find out what would happen to their volunteer buddies on stage. For their enthusiastic help, I rewarded the volunteers with "Atoms Family" coloring books from the American Nuclear Society, and wintergreen Lifesavers ${ }^{111}$ (which are triboluminescent).

To demonstrate Newton's first law, I had one volunteer sit in a chair on wheels and be Mr. Body. Another helper called Mr. Force gave Mr. Body a healthy push. As Newton predicted, Mr. Body kept rolling right out of view and he would have kept rolling had he not been acted on by the frictional force of the floor and his shoes. Newton's first law seemed more relevant and a little less threatening when the students saw that it is indeed related to many events in their lives.

To explain the second law of thermodynamics, I took a deck of cards that I had previously arranged in order of suit and rank. I showed the cards to the audience, shuffled them for several seconds, and gave them to a volunteer in the audience to put back in order. I asked him to call out when he was finished. I then continued with my presentation and a few minutes later when he returned the cards, everyone realized that it takes more energy to restore order than to make a mess. They more readily understood the statement that you can't clean up your room without making a mess somewhere else.

To tie all the laws together, I presented the Grand Unification of Physics, Thermodynamics, and Life. My objective was to show the students that failure is an important part of life. There are no safe, failure-free routes in life. We do learn more from our failures than our successes. I explained that $90 \%$ of research and development is failure but it is that $10 \%$ success that makes it all worthwhile. Most of the fear of failure

\section{Laws of Physics}

\#1. Everybody stays in a state of rest or in uniform motion unless acted upon by a force.

\#2 Force $=$ mass $\times$ acceleration

\#3 For every action there is an equal and opposite reaction.

\section{Laws of Thermodynamics}

\#1 All matter has energy, and energy is neither created nor destroyed.

\#2 Everything in the universe seeks its lowest level of energy.

\section{Grand Unification of Physics, Thermodynamics, and Life} Tom's Law of Relativity: All things are relative.

First Corollary-Life is difficult if all your relatives are successful. (No one is perfect!)

Second Corollary-Fear of failure is fear of change is fear of growing.

Third Corollary - Change is inherently good. Without it we'd still be swinging from trees! 
Timing is also crucial. I tried to cover Newton's laws before the students had studied them in class. Next time I will make my presentation after such material has been covered in class. Young minds show more interest in familiar things. I will also lecture less. My attempt to be philosophical was better suited to an older audience. In the future, to get my message across, I will use more events that the audience can relate to, as well as humor. But I will be sure that the humor is aimed at the specific audience. Sight gags work best during the demonstrations.

Last of all, I won't be surprised if I end up reaching only a small percentage of the audience. The stereotypical image, of the scientist has been with us for years. One lecture will not change that image but if we don't try, the scientific community will surely lose many bright minds to other "better advertised" professions. I'm looking forward to the next invitation to repeat my science commercial. I would welcome any comments or suggestions from readers to improve the next presentation.

T.C. Wiencek

T.C. Wiencek is an engineering specialist in the Mechanics of Materials Section of the Materials and Components Technology Division, Argonne National Laboratory. He can be contacted at Argonne National Laboratory, 9700 South Cass Avenue, Argonne, Illinois 60439, (708-252-5020). This work is supported by the U.S. Department of Energy, Office of Arms Control and Non-Proliferation, under Contract No. W-31-109-ENG-38.

The Education Exchange highlights the experiences of scientists and engineers with local schools, along with helpful hints and resources. If you would like to share your own involvement in science education, contact Finley Shapiro, Department of Electrical and Computer Engineering, Drexel University, Philadelphia, PA 19104, U.S.A.

Telephone (215) 895-6749

Fax (215) 895-1695

Email: shapiro@ece.drexel.edu

To receive additional information on how you can get involved in enhancing $\mathrm{K}-12$ science education, circle number 120 on the Reader Service Card.
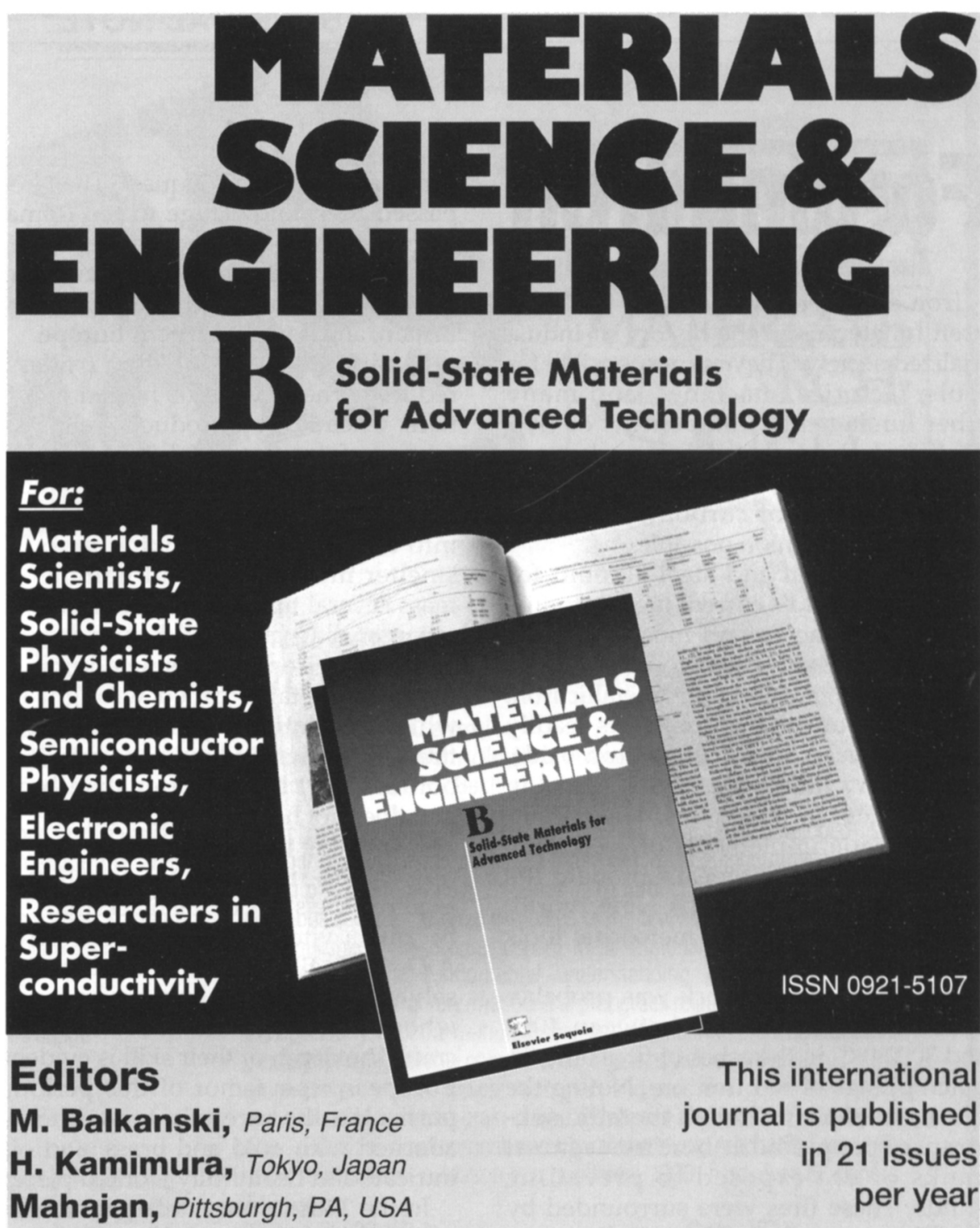

Materials Science \& Engineering B provides an international medium for the publication of theoretical and experimental studies and reviews related to the electronic, ionic, magnetic and optical properties of materials both in bulk and in thin films. Papers dealing with the processing, characterization, structure and physical properties of the crystalline, amorphous and glassy forms of metals, superconductors, semiconductors, insertion compounds, low-dimensional compounds and systems, fast-ion conductors, polymers and dielectrics are viewed as suitable for publication.

\section{ELSEVIER}

S E Q U O I A

PO Box 564

$\mathrm{CH}-1001$ Lausanne 1, Switzerland

Tel.: +41 (21) 207381

Fax: +41 (21) 232545

as of 14 September 1993

Tel.: +41 (21) 3207381

Fax: $+41(21) 3232545$
For customers in the USA and Canada.

Elsevier Science Publishing Co., Inc. Journal Information Center 655 Avenue of the Americas New York, NY 10010, USA Tel.: +1 (212) 633-3750 Fax: +1 (212) 633-3764
Please send a free sample copy and subscription information on

\section{MATERIALS SCIENCE \& ENGINEERING B}

Name:

Company/Institute:

Street: City:

Country: Postal Code:

Date: Signature: 